
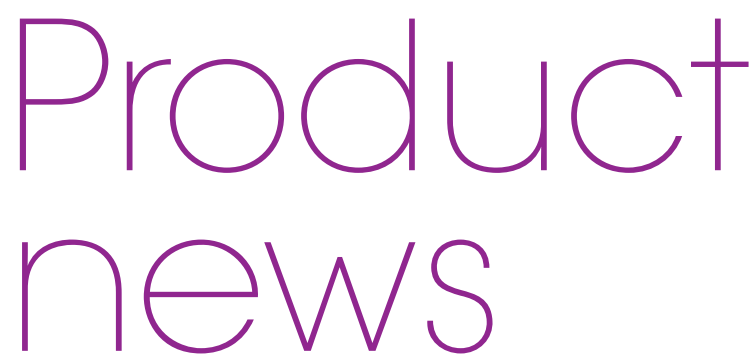

Product news is provided as a service to readers using text and images from

the manufacturer, supplier or distributor and does not imply endorsement

by BDJ Team. Normal and prudent research should be exercised before

purchase or use of any product mentioned.

\section{VISIT STAND BO3 AT THIS YEAR'S CONFERENCE}

This year the GSK Consumer Healthcare team will be visiting the British Dental Conference and Exhibition to showcase Sensodyne and Pronamel.

New Sensodyne Repair \& Protect - now with stronger repair - offers sensitivity sufferers the benefit of both advanced NovaMin technology and sodium fluoride in a single formulation. The immediate availability of sodium fluoride when brushing allows for greater fluoride uptake into the hydroxyapatite-like layer formed by NovaMin, over exposed dentine.

Pronamel toothpaste has an optimised fluoride formulation to help minerals penetrate deep into the tooth surface, actively strengthening and re-hardening acid-weakened enamel. Pronamel toothpaste delivers more fluoride deeper into enamel than other fluoride toothpaste helping to protect patients enamel from the effects of erosive tooth wear.

Visit stand B03 to try Sensodyne Repair \& Protect and Pronamel toothpaste for yourself in GSK's tasting station. The stand will also feature an interactive lecture revealing the truth about patients with dentine hypersensitivity and erosive tooth wear.

GSK are pleased to be sponsoring two presentations at the conference:

'Burden of Oral Diseases in Ageing Populations and links with General Health' - Georgios Tsakos, Thursday 26 May, 14:30-15:30

The presentation will review the epidemiological evidence on the burden of older adults and highlight the issues around the impact on quality of life.

'How to Manage and Prevent Patient Tooth Wear Caused by Dietary Acids' Rupert Austin, Friday 27 May, 11:45 - 12:45

The presentation will cover the clinical signs and symptoms of erosive tooth wear and dentine hypersensitivity as well as revealing the latest science on prevention and diagnosis of tooth wear and hypersensitivity.

For further information on Sensodyne and Pronamel visit stand B03 or www.gskdentalprofessionals.co.uk.

\section{LEARN ABOUT SUSTAINABLE DENTISTRY}

A new session on sustainable dentistry has been released on the award winning e-Learning for Healthcare (e-LfH) platform.

Released on NHS Sustainability Day 2016, the resource provides an introduction to climate change and explores the main principles of sustainable practice and their application in dentistry. It encourages clinicians and managers to make sustainable choices and identifies practical tools and resources to support this.

The material can be accessed by the dental team free of charge on the e-LfH website which provides $24 / 7$ access to nationally quality-assured materials on a wide range of topics in health and social care including dentistry. These sessions use an engaging and interactive presentation style using images, video and animation as well as self assessment to help build knowledge and understanding.

The e-learning resource has been developed by dentists Dr Devika Vadher and Dr Divya Verma, working with the Centre for Sustainable Healthcare (CSH) as Sustainable Dentistry Scholars - part-time roles funded by Health Education Kent, Surrey and Sussex.

http://www.e-lfh.org.uk/programmes/ dentistry/

www.sustainablehealthcare.org.uk

\section{SUPPORT YOUR DENTURE PATIENTS}

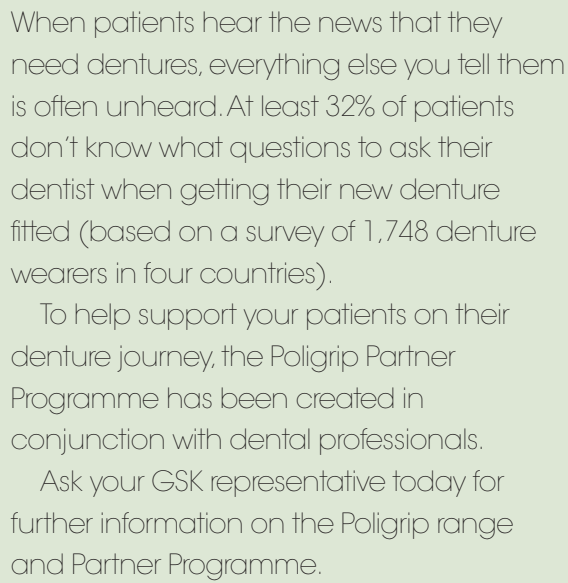

When patients hear the news that they need dentures, everything else you tell them is often unheard. At least $32 \%$ of patients don't know what questions to ask their dentist when getting their new denture fitted (based on a survey of 1,748 denture wearers in four countries)

To help support your patients on their denture journey, the Poligrip Partner Programme has been created in conjunction with dental professionals. Ask your GSK representative today for further information on the Poligrip range and Partner Programme.

The Poligrip range includes:

- Poligrip Overnight Whitening Daily Cleanser

- Poligrip 3 Minute Daily Cleanser

- Poligrip Ultra Fixative Cream

- Poligrip Flavour Free Fixative Cream.

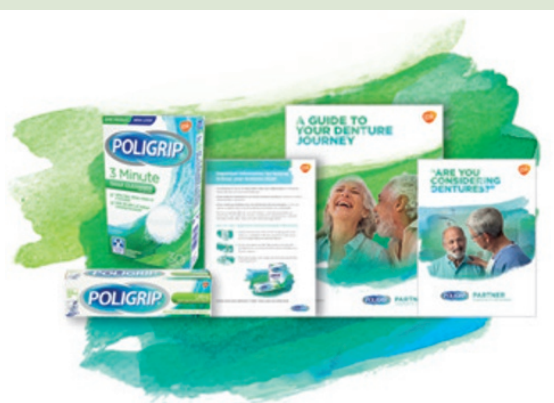

Recommend the Poligrip range to help your patients with dentures eat, speak and smile with confidence.

For additional support GSK have developed a distance learner module on the topic of 'Caring for Patients with Dentures'. The module has been designed to provide you with an insight into the patient experience of dentures as well as supporting you when providing practical advice

For further information on the Poligrip range and to complete the distance learner module visit wuw.gskdentalprofessionals.co.uk. Completion of the module can contribute up to 1.5 hours towards your verifiable CPD 